\title{
Multidimensional Aligned Nanowires Array: Toward Bendable and Stretchable Strain Sensors
}

\author{
Lili Meng ${ }^{\dagger}$, Jingchao Zhu ${ }^{\dagger}$, Ruixin Bian, Bojie Xu, Zhongxue Tang, Yu Zhang * and Huan Liu *
}

Key Laboratory of Bio-Inspired Smart Interfacial Science and Technology of Ministry of Education, School of Chemistry, Beihang University, Beijing 100191, China; mengll@buaa.edu.cn (L.M.); zhujingchao@buaa.edu.cn (J.Z.); bianrx785@buaa.edu.cn (R.B.); bojiexu@buaa.edu.cn (B.X.); by1727111@buaa.edu.cn (Z.T.)

* Correspondence: jade@buaa.edu.cn (Y.Z.); liuh@buaa.edu.cn (H.L.); Tel.: +86-010-82316066 (Y.Z.)

+ These two authors contributed equally to this work.

Citation: Meng, L.; Zhu, J.; Bian, R.; Xu, B.; Tang, Z.; Zhang, Y.; Liu, H. Multidimensional Aligned Nanowires Array: Toward Bendable and Stretchable Strain Sensors. Coatings 2021, 11, 975. https:// doi.org/10.3390/coatings11080975

Academic Editor: You Seung Rim

Received: 8 July 2021

Accepted: 12 August 2021

Published: 16 August 2021

Publisher's Note: MDPI stays neutral with regard to jurisdictional claims in published maps and institutional affiliations.

Copyright: (c) 2021 by the authors. Licensee MDPI, Basel, Switzerland. This article is an open access article distributed under the terms and conditions of the Creative Commons Attribution (CC BY) license (https:// creativecommons.org/licenses/by/ $4.0 /)$.
Abstract: Micropatterns based on the oriented nanowires have attracted research interests for their unique physicochemical advantages in various applications of electric microdevices. Here, we proposed a facile fibrous dewetting strategy by spreading and dewetting of the silver nanowire (AgNW) solution on the vertical aligned carbon nanotube array (ACNTs) for preparing multidimensional aligned nanowires array, based on the elastocapillary coalescence. The unidirectional shrinking of the liquid film on the top of ACNTs happens during the dewetting process, as a result of the elastocapillary coalescence of ACNTs, which drives the AgNWs aligned along normal direction of liquid film shrinkage on the top of ACNTs. Thus, a multidimensional aligned NWs array was prepared, composing of the horizontally oriented NWs of top layer and vertical ACNT bundles of under layer connected by CNT yarns. A bendable flexible electrode was prepared using the as-prepared multidimensional aligned nanowires array, showing high stability during bending cycles (1800 cycles). Moreover, the multidimensional aligned nanowires array is also applicable for fabricating strain sensors, which show stable resistance response under strain. We envision that the as-developed approach shed new light on easy manufacture NW-based micropatterns.

Keywords: nanowires; elastocapillary coalescence; dewetting; aligned; flexible sensor

\section{Introduction}

The nanowires-based micropatterns have provided vast opportunities for their unique physicochemical advantages in developing various photoelectric devices, including piezoelectric devices [1], sensing [2], light-emitting diodes [3], supercapacitors [4,5], and electronic skin [6,7]. In recent years, flexible sensors have attracted extensive research interests, which usually combine a conductive sensing element with a flexible supporting structure and enable transmuting deformation of the sensor into electric signals [8-10]. Various types of nanomaterials, such as carbon nanotubes [11,12], metal nanowires [13,14], graphene [15,16], and conductive polymers have been reported for making high performance sensor [17]. Among them, the nanowires-based sensors have aroused considerable interests due to the excellent electrical conductivity of one-dimensional (1D) nanomaterials. Moreover, the highly oriented nanowires (NWs) are especially desirable since they exhibit unique physicochemical advantage in terms of making high performance devices. So far, various solution-coating techniques have been developed for aligning 1D nanomaterials due for their merits of mild conditions and large-area preparation, such as printing [18], microfluidic approaches [19], rod coating [20,21], Langmuir-Blodgett [22-25], blade-coating $[26,27]$, and solution shearing technique $[28,29]$. However, these techniques suffer from complex for samples pretreatment process, uncontrolled deposition and aggregation of NWs during the film formation process, which remains a barrier for preparing the highly integrated devices. Particularly, preparing multidimensional oriented NWs 
array by one-step facile method remains a challenge. The essence of 1D nanomaterials arrangement is to introduce directional force during the film formation process, forcing 1D nanomaterials to arrange orderly. Recently, we developed multidimensional aligned NW array using fibrous elastocapillary coalescence of ACNTs that can align various NWs including $\mathrm{Ag}, \mathrm{ZnO}$, and $\mathrm{Al}_{2} \mathrm{O}_{3}$ [30]. The anisotropic shrinking of the liquid film on the top of ACNTs happens in this process requiring no external forces, which is an advantage for aligning NWs. It suggests that the fibrous elastocapillary coalescence can control the orientation of the NWs.

Here, we developed a facile strategy using fibrous elastocapillary coalescence of ACNTs for preparing multidimensional aligned nanowires array. The unidirectional shrinking of the liquid film on the top of ACNTs is enabled as a result of the elastocapillary coalescence of ACNTs. Consequently, multidimensional aligned nanowires array was developed, which is made up of three components: the horizontally aligned NWs of top layer, the vertical ACNT bundles of under layer and CNT yarns. The multidimensional ordered micropatterns could be used for the fabrication of flexible electrode and strain sensors, which show good stability against bending and strain.

\section{Materials and Methods}

Materials: Silver nanowires (AgNWs) solution with the diameter of $30 \pm 5 \mu \mathrm{m}$ and the diameter of $40 \pm 10 \mathrm{~nm}$ was acquired from Suzhou Cold Stones Technology Co., Ltd. (Suzhou, China). The ACNTs were purchased from Beijing Funate Innovation Technology Co., Ltd. (Beijing, China). Poly(ethylene terephthalate) (PET) with the thickness of $0.2 \mathrm{~mm}$ was obtained from Colleagues Hardware Co., Ltd. (Shanghai, China) poly(dimethylsiloxane) (PDMS) was purchased from Beijing Yinuokai Technology Co., Ltd. (Beijing, China).

Preparation of Multidimensional Aligned Nanowires Array: AgNWs were used dispersed in isopropyl alcohol with a concentration of $0.5 \mathrm{mg} \mathrm{mL}^{-1}$, which enables AgNWs to cover all areas of the vertically aligned carbon nanotube array [30]. The ACNTs with the area of $1 \times 1 \mathrm{~cm}^{2}$ and the height of $150 \mu \mathrm{m}$ were prepared by using chemical vapor deposition process, which involves the decomposition of xylene and ferrocene. Then, the ACNTs were transferred from glass substrates to PET and PDMS substrate, respectively, leading to the ACNTs with non-anchored sites. After that, the ACNTs on flexible substrate were dipped into AgNWs solution and then the ACNTs underwent a natural evaporation process at ambient condition without conducting any other management. Finally, the NWs array with multidimensional orientation were developed.

The Bending Test of the As-Prepared Multidimensional Aligned Nanowires Array: The electrical response of the multidimensional aligned NWs array on PET substrate was tested under the bending/unbending motion to evaluate electrical stability for the asprepared micropatterns with and without oriented AgNWs on top layer. The bending stability of the as-prepared NWs array was tested by recording the resistance change with numerous cycles of bending. The resistance change was denoted as the $R / R_{0}\left(R_{0}\right.$ is the original resistance of the micropatterns and $\mathrm{R}$ is the resistance on real time under the bending/unbending motion). The bending degree was described by $\left(L_{0}-L\right) / L_{0}\left(L_{0}\right.$ is the original length, $L$ is the length after bending), and the bending angle increase with enlarging the bending degree.

The Stretching Test of the As-Prepared Multidimensional Aligned NWs Array: The tensile stability of the multidimensional aligned NWs array on the PDMS substrate was monitored under stretching/ releasing cycles to evaluate the electrical stability of the asprepared NWs array. The resistance response of the multidimensional aligned NWs array on the PDMS substrate was monitored and recorded under stretching/releasing cycles at different strain degree.

Characterization: The structures of multidimensional aligned NWs array and the morphologies of the aligned AgNWs were characterized by a scanning electron microscope (S4800, HITACHI, Tokyo, Japan). The bending and stretching test for the as-prepared 
multidimensional aligned NWs array were conducted by a linear bending machine and the electrical signals were monitored at the same time by an electrochemical workstation (CHI660E, Shanghai Chenhua Instrument Co., LTD., Shanghai, China).

\section{Results and Discussions}

The fabrication of multidimensional aligned NWs array guided by the fibrous elastocapillary coalescence was schematically shown in Figure 1 . The ACNTs (height of $\approx 150 \mu \mathrm{m}$ ) arranged vertically on the glass substrate were particularly used (Figure 1a). Then, the ACNTs were transferred from glass substrates to flexible substrate (PET, PDMS), leading to the ACNTs with non-anchored sites (Figure 1b). Isopropyl alcohol was used as the solvent. The surface tension of isopropyl alcohol is as low as $21.7 \mathrm{mN} \mathrm{m}^{-1}$, which endowed the fast-dewetting ability on the ACNTs. The ACNTs on flexible substrate were dipped into AgNWs solution and then the ACNTs underwent a natural evaporation process at ambient condition without conducting any other management (Figure 1c,d). As we previously suggested, elastocapillary coalescence occurred with the solvent evaporates, which causes strong capillary forces, interfacial tension, and van der Waals interactions, leading to aggregating and bundling of the ACNTs. As a result, multidimensional aligned NWs array with a typical polygon-shape structure formed, as illustrated in Figure 1e. Note that the bundling of the ACNTs facilitates directional shrinking of the liquid film on the top of the ACNTs, making the randomly distributed NWs move and rotate into alignment. With the bundling of the ACNTs, CNT yarns were pulled out connecting adjacent ACNT bundles. Ultimately, NWs array with multidimensional orientation was developed, which is made up of three components: the horizontally aligned NWs of top layer, the vertical ACNT bundles of under layer and CNT yarns.

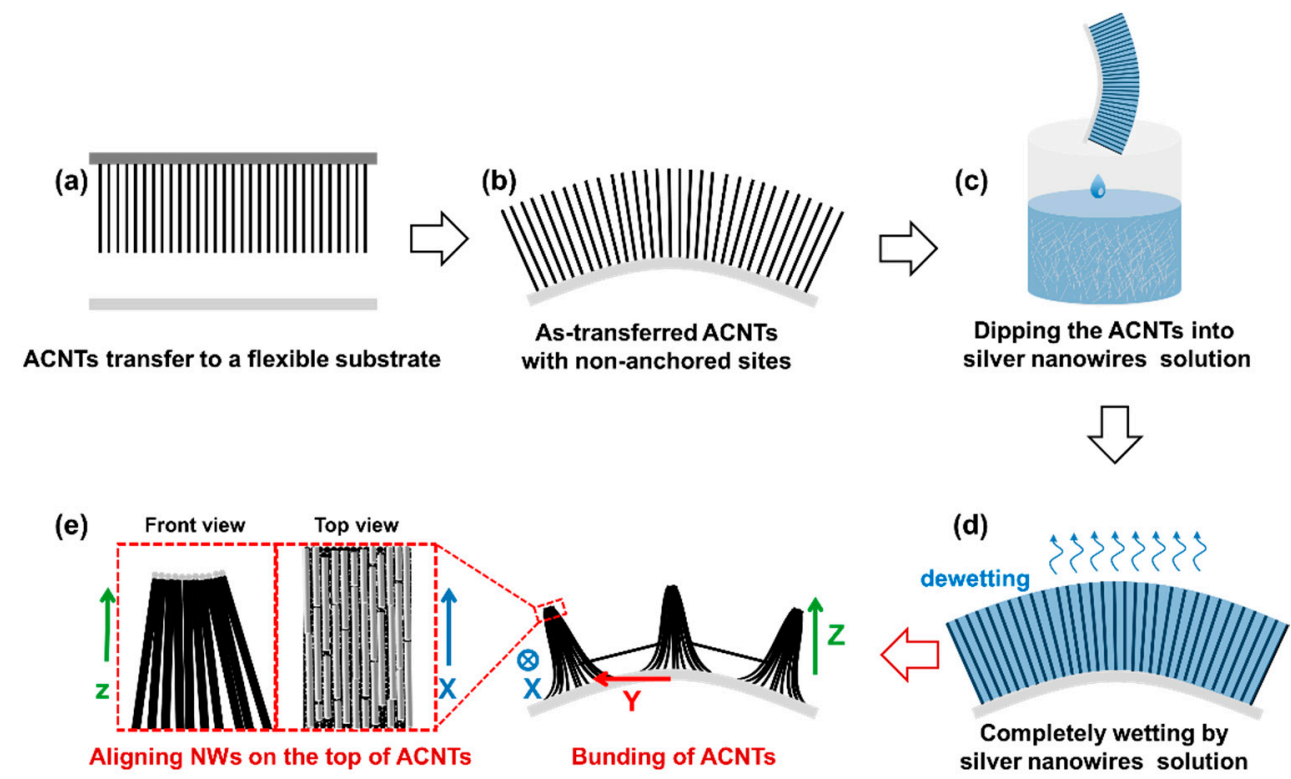

Figure 1. Schematic depiction for the formation process of the multidimensional aligned NWs array. (a) ACNTs were transferred from glass substrates to flexible substrates. (b) ACNTs on flexible substrates. (c) The ACNTs on the flexible substrates were dipped into AgNWs solution. (d) The ACNTs underwent a natural evaporation process at ambient condition without conducting any other management. (e) As the solution evaporates, aggregating and bundling of the ACNTs happens because of the elastocapillary coalescence, based on which the multidimensional aligned NWs array were developed. The AgNWs on the top layer of the ACNTs were aligned.

The SEM observation of the as-developed multidimensional aligned NWs array was conducted. As shown, a typical polygon-shape structure was observed. This is due to the elastocapillary coalescence during the dewetting process of the solution on the densely 
distributed ACNTs, leading to the bundling of CNTs (Figure 2a). Here, $x, y$, and $z$ indicate direction of the oriented NWs of top layer, CNT yarns, and bundled ACNTs of under layer, respectively. The enlarged SEM image of the top-layer NWs show that NWs were highly aligned along the $x$-direction (Figure $2 b$ ). The CNT yarns were clearly seen in polygonshape structure, which is oriented in certain direction (Figure 2c). The morphology of under-layer ACNTs were further observed, indicating the orientation in the z-direction (Figure 2d).

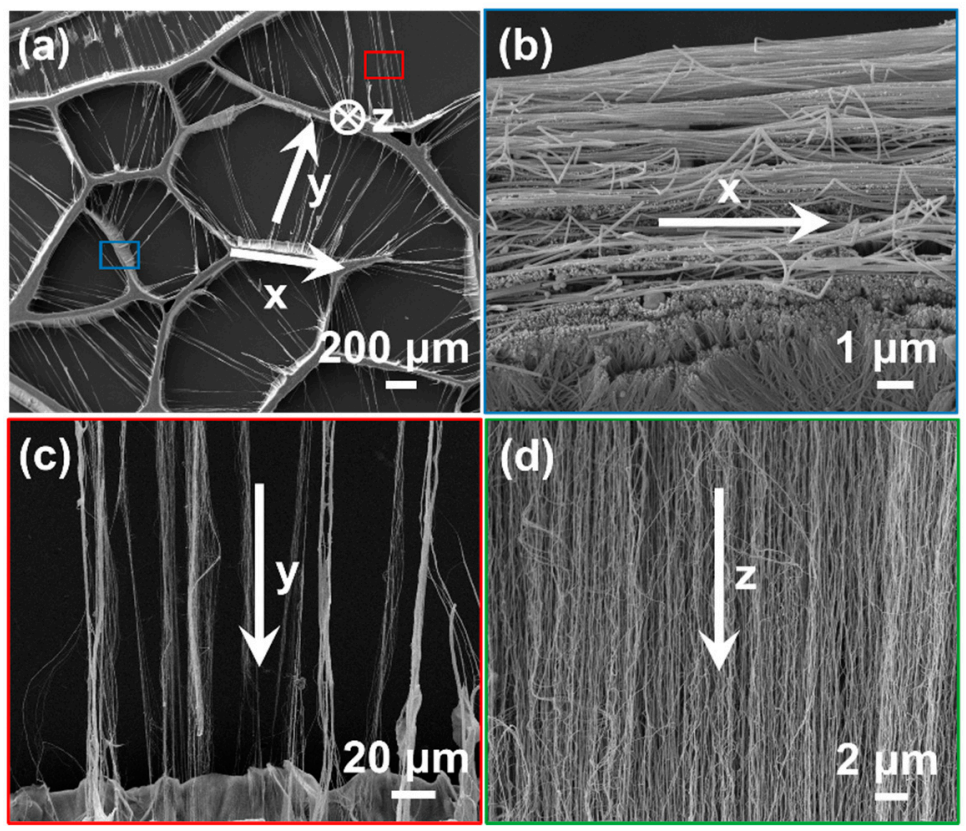

Figure 2. SEM images of the as-prepared NWs array with multidimensional orientation. (a) The top-view SEM image of the multidimensional aligned NWs array, exhibiting a typical polygon-shape structure. The SEM image reveal that the horizontally oriented NWs, CNT yarns in x, y direction, vertically aligned ACNTs in z direction, respectively. (b) The SEM images of top-layer oriented AgNWs. (c) The SEM image of CNT yarns in y direction. (d) The SEM images of under-layer bundled ACNTs in $\mathrm{z}$ directions.

The as-prepared multidimensional aligned NWs array can be used as flexible electrodes. To evaluate its mechanical stability, the responses of electric resistance to mechanical deformations under bending/unbending motion were investigated (Figure 3). For comparison, the resistance changes for the micropatterns with and without oriented NWs on top layer (Figure 3b,c) were tested (Figure 3). For the NWs array with oriented NWs on top layer, the electrical response exhibited high stability during bending and unbending cycles. As shown in Figure $3 b_{1}$, the resistance linearly increases with the bending degree enlarging and there was almost no change after it was released. Even after 1800 bending/unbending cycles under bending degree of $10 \%$, very limited increase for the resistance of the NWs array with oriented NWs on top layer was observed (Figure 3b, black line). However, for the multidimensional aligned NWs array without oriented NWs on top layer, a disruption of the electrical signals was observed after 750 bending/unbending cycles. This indicated that the multidimensional aligned NWs array with oriented NWs on top layer show excellent mechanical stability under bending stress. To investigate the reason of the better bending stability of the micropattern with oriented NWs on top-layer than that of the without oriented NWs on top-layer, the SEM observation on both NWs array was conducted after bending test. SEM image of the NWs array with oriented NWs on top layer shows negligible changes of morphology after reciprocating bending cycles, indicating the stability of the NWs array as a flexible electrode (Figure 3c). However, the NWs array without oriented NWs on top layer are liable to be broke under the bending stress (Figure 3d). 
The multidimensional aligned NWs array based flexible electrodes showed quite stable and reliable response to the applied bending strain. Figure $3 e$ shows the relative resistance change of as-prepared NWs array flexible electrodes under cyclic bending-unbending of different bending degree with the same frequency. The relative resistance increases with the bending degree enhancing. Taken together, the oriented NWs on top-layer helps build up the resistance to the external bending, which results in the excellent mechanical stability.

(a)

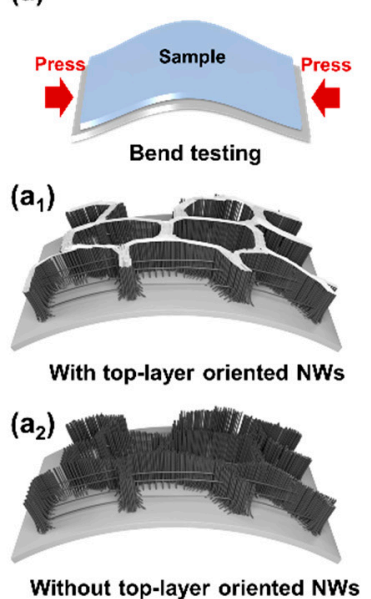

(c)

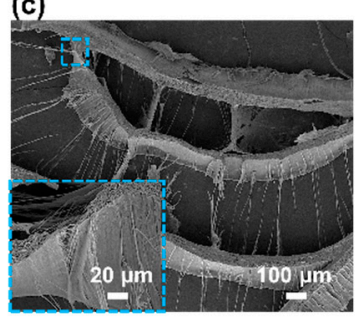

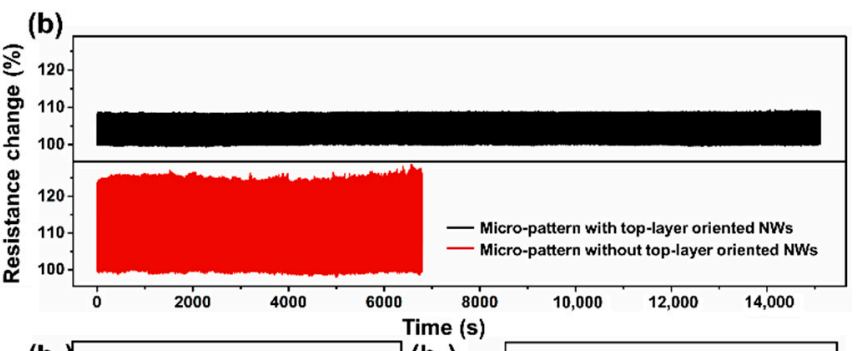

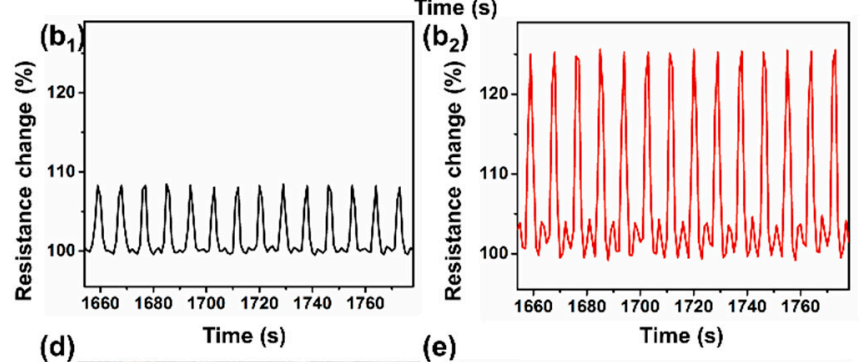

(d)

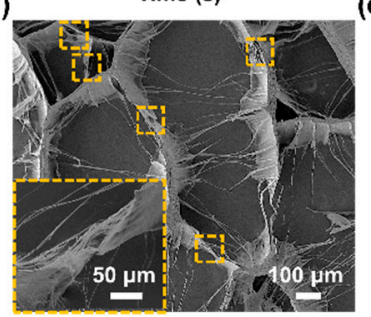

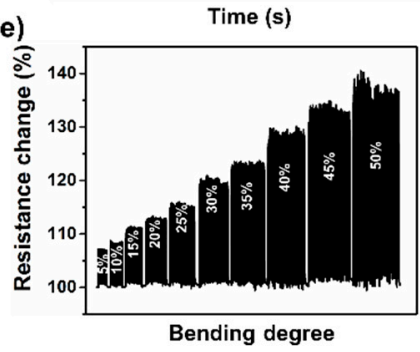

Figure 3. Bending stability of the as-prepared multidimensional aligned NWs array. (a) Schematic illustration of the testing process. $\left(\mathbf{a}_{1}, \mathbf{a}_{2}\right)$ The NWs array with and without oriented AgNWs on top layer. (b) The resistance changes of the NWs array with and without top-layer oriented AgNWs under bending/unbending motion at bending degree of $10 \%$. $\left(\mathbf{b}_{1}, \mathbf{b}_{2}\right)$ An enlarged picture of $(\mathbf{b})$ curves for the NWs array with and without top-layer oriented AgNWs, respectively. (c,d) The SEM picture of the NWs array with and without oriented NWs on the top-layer after 750 bending cycles. The typic enlarged view was shown in the illustration. (e) The resistance changes of the as-prepared multidimensional aligned NWs array under bending/unbending motion at different bending degree of $5 \%, 10 \%, 15 \%, 20 \%, 25 \%, 30 \%, 35 \%, 40 \%, 45 \%$, and $50 \%$, respectively.

We also demonstrated a strain sensor using the as-developed multidimensional aligned NWs array, as Figure 4 illustrates. Stretchability is a very important characteristic for flexible electrodes. Hence, we conducted tensile stretching test of the multidimensional aligned NWs array electrodes with monitoring the resistance (Figure 4a). The as-prepared strain sensors based on the NWs array with oriented NWs on top layer showed long-time stability, high durability (Figure $4 \mathrm{~b}$ ). As shown in Figure $4 \mathrm{~b}_{1}$, during each period, the resistance of the NWs array with oriented NWs on top-layer linearly increased as the strain enlarging. Figure $4 \mathrm{~b}$ (black line) shows the response of strain sensor to 800 times cyclic loading of $10 \%$ strain, indicating its remarkable stability. SEM observation of NWs array with oriented NWs on top-layer was conducted after stretching-releasing cycle, confirming that the stretching motion led to negligible cracks. However, for the NWs array without oriented NWs on top-layer, the sheet resistance exhibited unstable response under stretching-releasing motions and eventually failed at a low number of cycles $(<300)$. This is due to the undesired cracks happening on the NWs array without oriented NWs on top 
layer during the stretching-releasing (Figure 4d). Figure 4e shows the resistance change of a strain sensor based on the as-prepared NWs array with oriented NWs on top layer under cyclic stretching-releasing of different strains with the same frequency. The relative resistance changes increased as the strain increased. Taken together, the results indicated that the top-layer oriented NWs enhance the mechanical properties of the micropatterns, leading to the improvement of the stability of the micropatterns.

(a)

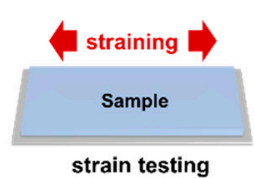

$\left(a_{1}\right)$

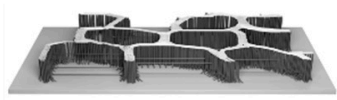

With top-layer oriented NWs

$\left(a_{2}\right)$

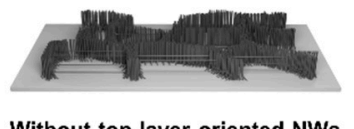

(c)

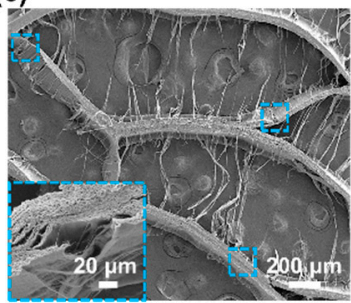

(b)
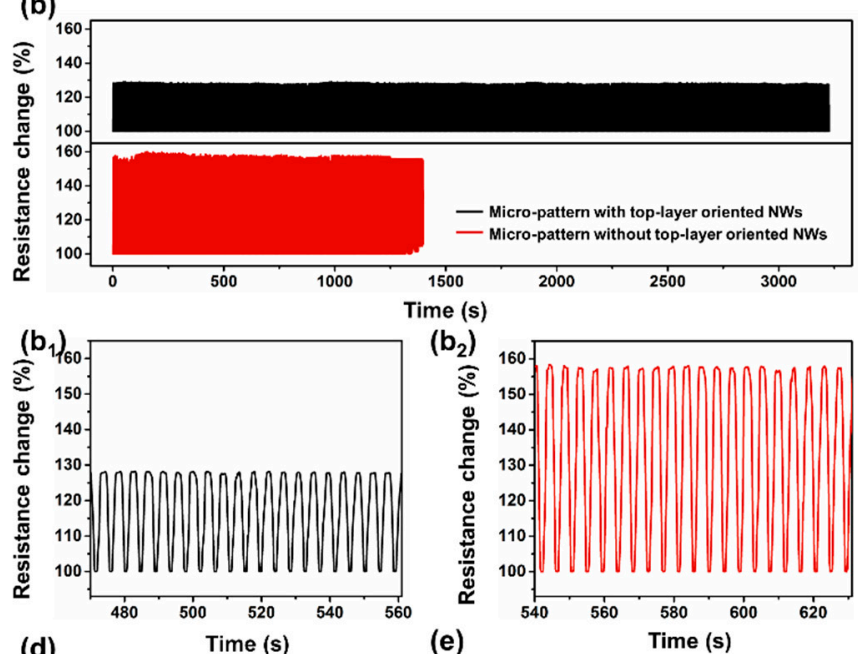

$\left(b_{2}\right)$

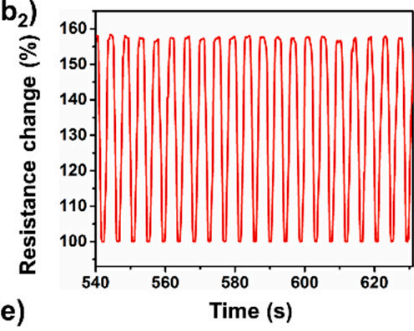

(e)

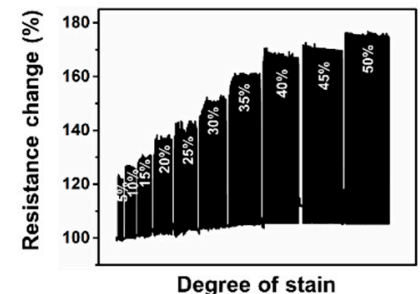

Figure 4. The performance of the multidimensional aligned NWs array-based sensor. (a) Schematic depiction for the stretching process. $\left(\mathbf{a}_{1}, \mathbf{a}_{2}\right)$ A schematic of multidimensional aligned NWs array with and without oriented NWs on top layer as strain sensor. (b) Change in resistance under stretching/releasing motion of $10 \%$ strain for 300 cycles, showing the durability of the NWs array with top-layer oriented NWs. $\left(\mathbf{b}_{1}, \mathbf{b}_{2}\right)$ The typic enlarged view of Figure $4 b$ for the NWs array with and without oriented AgNWs on top layer, respectively. (c,d) The SEM image of NWs array with and without oriented NWs on the top layer after 300 stretching cycles. The insert shows the local enlarged picture. (e) The electrical response of the as-prepared multidimensional aligned NWs array under stretching/releasing motion at different strain degree of 5\%, 10\%, 15\%, 20\%, 25\%, 30\%, 35\%, $40 \%, 45 \%$, and $50 \%$, respectively.

\section{Conclusions}

In summary, we proposed a facile method using fibrous elastocapillary coalescence of ACNTs for preparing multidimensional aligned nanowires array. With the elastocapillary coalescence of ACNTs, the unidirectional shrinking of the liquid film on the top of ACNTs happens in this process requiring no external forces, leading to a multidimensional aligned nanowires array composed of the horizontally oriented NWs of top layer and the vertical ACNT bundles of under layer and CNT yarns that act as a link. Based on the as-prepared multidimensional aligned nanowires array, a bendable flexible electrode was prepared, which show high stability during bending cycles (1800 cycles). Moreover, we also demonstrated that the multidimensional aligned nanowires array is applicable for making a strain sensor, which show stable resistance response under strain. The result offers new applications in fabricating NW-based micropatterns applied in bendable and stretchable electric devices. 
Author Contributions: Data curation, L.M., J.Z., R.B. and B.X.; Formal analysis, L.M., J.Z., R.B. and Z.T.; Project administration, H.L.; Resources, H.L.; Supervision, H.L.; Writing一original draft, L.M. and J.Z.; Writing-review and editing, Y.Z. and H.L. All authors have read and agreed to the published version of the manuscript.

Funding: This work was financially supported by the National Key R\&D Program of China (No. 2018YFA0704801) and the National Natural Science Foundation of China (No. 21872002).

Institutional Review Board Statement: Not applicable.

Informed Consent Statement: Not applicable.

Data Availability Statement: The associated data in this study are available from the corresponding author.

Acknowledgments: The authors are grateful to Mingzhe Shao for the assistance in scanning electron microscope measurements.

Conflicts of Interest: The authors declare no conflict of interest.

\section{References}

1. Persano, L.; Dagdeviren, C.; Su, Y.; Zhang, Y.; Girardo, S.; Pisignano, D.; Huang, Y.; Rogers, J.A. High performance piezoelectric devices based on aligned arrays of nanofibers of poly (vinylidenefluoride-co-trifluoroethylene). Nat. Commun. 2013, 4, 1633. [CrossRef]

2. Fennell, J.F.; Liu, S.F.; Azzarelli, J.M.; Weis, J.G.; Rochat, S.; Mirica, K.A.; Ravnsbæk, J.B.; Swager, T.M. Nanowire chemical/biological sensors: Status and a roadmap for the future. Angew. Chem. Int. Ed. 2016, 55, 1266-1281. [CrossRef]

3. Zhou, L.; Xiang, H.Y.; Shen, S.; Li, Y.Q.; Chen, J.D.; Xie, H.J.; Goldthorpe, I.A.; Chen, L.S.; Lee, S.T.; Tang, J.X. High-performance flexible organic light-emitting diodes using embedded silver network transparent electrodes. ACS Nano 2014, 8, 12796-12805. [CrossRef]

4. Chen, S.; Shi, B.; He, W.; Wu, X.; Zhang, X.; Zhu, Y.; He, S.; Peng, H.; Jiang, Y.; Gao, X.; et al. Quasifractal networks as current collectors for transparent flexible supercapacitors. Adv. Funct. Mater. 2019, 29, 1906618. [CrossRef]

5. Liu, T.; Yan, R.; Huang, H.; Pan, L.; Cao, X.; DeMello, A.; Niederberger, M. A Micromolding Method for Transparent and Flexible Thin-Film Supercapacitors and Hybrid Supercapacitors. Adv. Funct. Mater. 2020, 30, 2004410. [CrossRef]

6. Wang, S.; Xu, J.; Wang, W.; Wang, G.J.N.; Rastak, R.; Molina-Lopez, F.; Chung, J.W.; Niu, S.; Feig, V.R.; Lopez, J.; et al. Skin electronics from scalable fabrication of an intrinsically stretchable transistor array. Nature 2018, 555, 83-88. [CrossRef] [PubMed]

7. Lipomi, D.J.; Vosgueritchian, M.; Tee, B.C.K.; Hellstrom, S.L.; Lee, J.A.; Fox, C.H.; Bao, Z. Skin-like pressure and strain sensors based on transparent elastic films of carbon nanotubes. Nat. Nanotechnol. 2011, 6, 788-792. [CrossRef]

8. Xia, K.; Jian, M.; Zhang, Y. Advances in wearable and flexible conductors based on nanocarbon materials. Acta Phys. Chim. Sin. 2016, 32, 2427-2446. [CrossRef]

9. Amjadi, M.; Kyung, K.U.; Park, I.; Sitti, M. Stretchable, skin-mountable, and wearable strain sensors and their potential applications: A review. Adv. Funct. Mater. 2016, 26, 1678-1698. [CrossRef]

10. Park, S.; Vosguerichian, M.; Bao, Z. A review of fabrication and applications of carbon nanotube film-based flexible electronics. Nanoscale 2013, 5, 1727-1752. [CrossRef]

11. Ryu, S.; Lee, P.; Chou, J.B.; Xu, R.; Zhao, R.; Hart, A.J.; Kim, S.G. Extremely elastic wearable carbon nanotube fiber strain sensor for monitoring of human motion. ACS Nano 2015, 9, 5929-5936. [CrossRef]

12. Liu, Z.; Qi, D.; Guo, P.; Liu, Y.; Zhu, B.; Yang, H.; Chen, X. Thickness-gradient films for high gauge factor stretchable strain sensors. Adv. Mater. 2015, 27, 6230-6237. [CrossRef]

13. Araki, T.; Uemura, T.; Yoshimoto, S.; Takemoto, A.; Noda, Y.; Izumi, S.; Sekitani, T. Wireless monitoring using a stretchable and transparent sensor sheet containing metal nanowires. Adv. Mater. 2020, 32, 1902684. [CrossRef]

14. Zhang, L.; Li, H.; Lai, X.; Gao, T.; Zeng, X. Three-dimensional binary-conductive-network silver nanowires@ thiolated graphene foam-based room-temperature self-healable strain sensor for human motion detection. ACS Appl. Mater. Interfaces 2020, 12, 44360-44370. [CrossRef] [PubMed]

15. Cheng, Y.; Wang, R.; Sun, J.; Gao, L. A stretchable and highly sensitive graphene-based fiber for sensing tensile strain, bending, and torsion. Adv. Mater. 2015, 27, 7365-7371. [CrossRef] [PubMed]

16. Park, J.J.; Hyun, W.J.; Mun, S.C.; Park, Y.T.; Park, O.O. Highly stretchable and wearable graphene strain sensors with controllable sensitivity for human motion monitoring. ACS Appl. Mater. Interfaces 2015, 7, 6317-6324. [CrossRef] [PubMed]

17. Zamiri, G.; Haseeb, A.S.M.A. Recent trends and developments in graphene/conducting polymer nanocomposites chemiresistive sensors. Materials 2020, 13, 3311. [CrossRef] [PubMed]

18. Kang, S.; Kim, T.; Cho, S.; Lee, Y.; Choe, A.; Walker, B.; Ko, S.J.; Kim, J.Y.; Ko, H. Capillary Printing of Highly Aligned Silver Nanowire Transparent Electrodes for High-Performance Optoelectronic Devices. Nano Lett. 2015, 15, 7933-7942. [CrossRef]

19. Lee, P.; Lin, R.; Moon, J.; Lee, L.P. Microfluidic alignment of collagen fibers for in vitro cell culture. Biomed. Microdevices. 2006, 8 , 35-41. [CrossRef] 
20. Sun, Y.; Chang, M.; Meng, L.; Wan, X.; Gao, H.; Zhang, Y.; Zhao, K.; Sun, Z.; Li, C.; Liu, S.; et al. Flexible Organic Photovoltaics Based on Water-Processed Silver Nanowire Electrodes. Nat. Eletron. 2019, 2, 513-520. [CrossRef]

21. Cho, S.; Kang, S.; Pandya, A.; Shanker, R.; Khan, Z.; Lee, Y.; Park, J.; Craig, S.L.; Ko, H. Large-Area Cross-Aligned Silver Nanowire Electrodes for Flexible, Transparent, and Force-Sensitive Mechanochromic Touch Screens. ACS Nano 2017, 11, $4346-4357$. [CrossRef]

22. Wang, J.; Lu, Y.; Li, H.; Liu, J.; Yu, S. Large Area Co-Assembly of Nanowires for Flexible Transparent Smart Windows. J. Am. Chem. Soc. 2017, 139, 9921-9926. [CrossRef]

23. Bian, R.; Meng, L.; Zhang, M.; Chen, L.; Liu, H. Aligning one-dimensional nanomaterials by solution processes. ACS Omega 2019, 4, 1816-1823. [CrossRef]

24. Li, X.; Zhang, L.; Wang, X.; Shimoyama, I.; Sun, X.; Seo, W.S.; Dai, H. Langmuir-Blodgett assembly of densely aligned single-walled carbon nanotubes from bulk materials. J. Am. Chem. Soc. 2007, 129, 4890-4891. [CrossRef]

25. Lv, J.; Hou, K.; Ding, D.; Wang, D.; Han, B.; Gao, X.; Zhao, M.; Shi, L.; Guo, J.; Zheng, Y.; et al. Gold nanowire chiral ultrathin films with ultrastrong and broadband optical activity. Angew. Chem. Int. Ed. 2017, 56, 5055-5060. [CrossRef]

26. Ko, Y.; Song, S.K.; Kim, N.H.; Chang, S.T. Highly Transparent and Stretchable Conductors Based on a Directional Arrangement of Silver Nanowires by a Microliter-Scale Solution Process. Langmuir 2016, 32, 366-373. [CrossRef]

27. Fang, Y.; Ding, K.; Wu, Z.; Chen, H.; Li, W.; Zhao, S.; Zhang, Y.; Wang, L.; Zhou, J.; Hu, B. Architectural engineering of nanowire network fine pattern for $30 \mu \mathrm{m}$ wide flexible quantum dot light-emitting diode application. ACS Nano 2016, 10, 10023-10030. [CrossRef] [PubMed]

28. Worfolk, B.J.; Andrews, S.C.; Park, S.; Reinspach, J.; Liu, N.; Toney, M.F.; Mannsfeld, S.C.; Bao, Z. Ultrahigh electrical conductivity in solution-sheared polymeric transparent films. Proc. Natl. Acad. Sci. USA 2015, 112, 14138-14143. [CrossRef] [PubMed]

29. Park, S.; Pitner, G.; Giri, G.; Koo, J.H.; Park, J.; Kim, K.; Wang, H.; Sinclair, R.; Wong, H.S.P.; Bao, Z. Large-Area Assembly of Densely Aligned Single-Walled Carbon Nanotubes Using Solution Shearing and Their Application to Field-Effect Transistors. Adv. Mater. 2015, 27, 2656-2662. [CrossRef]

30. Bian, R.; Meng, L.; Guo, C.; Tang, Z.; Liu, H. A Facile One-Step Approach for Constructing Multidimensional Ordered Nanowire Micropatterns via Fibrous Elastocapillary Coalescence. Adv. Mater. 2019, 31, 1900534. [CrossRef] [PubMed] 